\title{
OPTIMAL CONTROL OF AN ILL-POSED ELLIPTIC SEMILINEAR EQUATION WITH AN EXPONENTIAL NON LINEARITY
}

\author{
E. CASAS, O. KAVIAN, AND J.-P. PUEL
}

\begin{abstract}
We study here an optimal control problem for a semilinear elliptic equation with an exponential nonlinearity, such that we cannot expect to have a solution of the state equation for any given control. We then have to speak of pairs (control, state). After having defined a suitable functional class in which we look for solutions, we prove existence of an optimal pair for a large class of cost functions using a non standard compactness argument. Then, we derive a first order optimality system assuming the optimal pair is slightly more regular.
\end{abstract}

\section{INTRODUCTION}

In this paper we are concerned with the optimal control of the following semilinear elliptic equation

$$
\begin{cases}-\Delta y=\mathrm{e}^{y}+u & \text { in } \Omega, \\ y=0 & \text { on } \Gamma,\end{cases}
$$

where $\Omega \subset \mathbb{R}^{n}, n>2$, is a bounded open set, $\Gamma$ being the boundary, which is assumed to be Lipschitz. The function $u$ is the control, that will be taken in some space $L^{p}(\Omega)$, and $y$ denotes the state in our control problem.

The equation (1.1) appears in several contexts: we refer for instance to D.A. Franck-Kamenetskii [5] for combustion theory in chemical reactors, S. Chandrasekhar [3] in the study of stellar structures. The equation (1.1) is ill-posed in the sense that there is no solution for some controls $u$ and many solutions can be found for some others (see for instance I.M. Gelfand [7], M.G. Crandall and P.H. Rabinowitz [4], F. Mignot and J.P. Puel [8, 9], Th. Gallouët, F. Mignot and J.P. Puel [6]). Because of the term $\mathrm{e}^{y}$, we need

E. Casas: Dpt. Matemática Aplicada Y Ciencias de la Computación, E.T.S.I.I y T., Univiersidad de Cantabria, Av. Los Castros s/n 39005 Santander, Spain. E-mail: casasetsiso.macc.unican.es.

O. Kavian: Université de Versailles Saint-Quentin et Centre de Mathématiques Appliquées, Ecole Polytechnique, 91128 Palaiseau Cedex, France. E-mail: kavian@math.uvsq.fr.

J.-P. Puel: Université de Versailles Saint-Quentin et Centre de Mathématiques Appliquées, Ecole Polytechnique, 91128 Palaiseau Cedex, France. E-mail: jppuel ocmapx . polytechnique.fr.

This research was partially supported by the European Union, under HCM Project number ERBCHRXCT940471. The first author was also partially supported by Dirección General de Investigación Científica y Técnica (Spain).

Received by the journal October 28, 1997. Revised July 6, 1998. Accepted for publication July 16, 1998.

(C) Société de Mathématiques Appliquées et Industrielles. Typeset by IATEX. 
to explain what we mean by a solution of (1.1). We will say that $y$ is a solution of (1.1) if it belongs to the class of functions

$$
Y=\left\{y \in H_{0}^{1}(\Omega): \mathrm{e}^{y} \in L^{1}(\Omega)\right\}
$$

and it satisfies the equation in the distribution sense. Then the optimal control problem will be formulated in the following terms

$$
\text { (P) }\left\{\begin{array}{l}
\text { Minimize } J(y, u):=\int_{\Omega} L(x, y(x)) d x+\frac{N}{p} \int_{\Omega}|u(x)|^{p} d x \\
(y, u) \in Y \times K \text { satisfies }(1.1),
\end{array}\right.
$$

where $K$ is a nonempty closed convex subset of $L^{p}(\Omega), 2 \leq p<+\infty, N \geq 0$ and $L: \Omega \times \mathbb{R} \longrightarrow \mathbb{R}$ is a Carathéodory function of class $C^{1}$ with respect to the second variable and satisfying appropriate growth conditions which will be shown to be the following

$$
\begin{aligned}
\left|\frac{\partial L}{\partial y}(x, y)\right| & \leq a_{1}(x)+\alpha_{1}\left(\left|y^{-}\right|^{\theta_{1}}+\mathrm{e}^{y}\right), \\
L(x, y) & \geq a_{2}(x)-\alpha_{2}\left(\left|y^{-}\right|^{\theta_{2}}+\mathrm{e}^{y}\right),
\end{aligned}
$$

for some $a_{1}, a_{2} \in L^{1}(\Omega), \alpha_{1}, \alpha_{2} \geq 0, \theta_{1}=n p /(n-2 p)$ if $p<n / 2$, and $0 \leq \theta_{1}<+\infty$ if $p \geq n / 2$, and $1 \leq \theta_{2}<p$. For instance, $y_{d} \in Y$ being given, a typical functional $J$ would be

$$
J(y, u):=\frac{1}{2} \int_{\Omega}\left|y(x)-y_{d}(x)\right|^{2} d x+\frac{N}{p} \int_{\Omega}|u(x)|^{p} d x .
$$

We should emphasize on the fact that one of the main difficulties of the problem is to choose an appropriate class of solutions such that $(\mathrm{P})$ has a solution in that class.

The plan of the paper is as follows. In Section 2 we will analyze the state equation and we will establish the necessary background to study the control problem. The existence of a solution for $(\mathrm{P})$ is studied in Sections 3 and 4 for the cases $p>2$ and $p=2$, respectively. The case $p=2$ presents some difficulties and we will be able to prove the existence of an optimal control under some additional assumption on the function $L$. We should note that, as it seems to us, the case $1 \leq p<2$ cannot be treated with the techniques we use in this paper. Finally in Section 5 the optimality conditions will be investigated.

\section{Analysis of the State Equation}

We start this section by establishing that any solution of (1.1) in the sense defined in Section 1 is a solution in the variational sense in $H_{0}^{1}(\Omega)$; this requires to prove some regularity of the term $\mathrm{e}^{y}$.

Lemma 2.1. Let $y \in Y$ be a solution of (1.1), then $\mathrm{e}^{y} \in H^{-1}(\Omega)$, $\mathrm{e}^{y} z \in$ $L^{1}(\Omega)$ for every $z \in H_{0}^{1}(\Omega)$ and

$$
\int_{\Omega} \nabla y(x) \nabla z(x) d x=\int_{\Omega}\left[\mathrm{e}^{y(x)}+u(x)\right] z(x) d x .
$$


Proof. By the definition of a solution of (1.1), for all $z \in C_{c}^{\infty}(\Omega)$ we have

$$
\int_{\Omega} \nabla y(x) \nabla z(x) d x=\int_{\Omega}\left[\mathrm{e}^{y(x)}+u(x)\right] z(x) d x .
$$

Given $z \in L^{\infty}(\Omega) \cap H_{0}^{1}(\Omega)$, we can take a sequence $\left\{z_{k}\right\}_{k=1}^{\infty} \subset C_{c}^{\infty}(\Omega)$, with $\left\|z_{k}\right\|_{\infty} \leq\|z\|_{\infty}+1$, for every $k \in \mathbb{N}$ and $z_{k} \rightarrow z$ in $H_{0}^{1}(\Omega)$ and $z_{k} \rightarrow z$ in $L^{\infty}(\Omega)-\mathrm{W}^{*}$. Then for all $k \geq 1$ we can replace $z$ by $z_{k}$ in $(2.2)$ and pass to the limit when $k \rightarrow \infty$ to obtain that the identity in (2.2) is also true for any $z \in L^{\infty}(\Omega) \cap H_{0}^{1}(\Omega)$.

Let us take now $z \in H_{0}^{1}(\Omega)$ such that $z \geq 0$ and set

$$
T_{k}(z)(x):= \begin{cases}k & \text { if } z(x)>k \\ z(x) & \text { if } 0 \leq z(x) \leq k\end{cases}
$$

Then $T_{k}(z) \in L^{\infty}(\Omega) \cap H_{0}^{1}(\Omega)$ and

$$
\int_{\Omega} \nabla y(x) \nabla T_{k}(z)(x) d x=\int_{\Omega}\left[\mathrm{e}^{y(x)}+u(x)\right] T_{k}(z)(x) d x \quad \forall k \in \mathbb{N} .
$$

Since $T_{k}(z) \rightarrow z$ in $H_{0}^{1}(\Omega)$, the only trouble to pass to the limit in this identity comes from the term $\mathrm{e}^{y} z_{k}$. As $T_{k}(z) \geq 0$ and $\mathrm{e}^{y}>0$, then from the monotone convergence theorem, taking into account that $T_{k}(z)(x) \uparrow z(x)$ for almost all $x \in \Omega$, we deduce

$$
\begin{aligned}
\int_{\Omega} \mathrm{e}^{y(x)} z(x) d x & =\lim _{k \rightarrow+\infty} \int_{\Omega} \mathrm{e}^{y(x)} T_{k}(z)(x) d x \\
& =\lim _{k \rightarrow+\infty}\left\{\int_{\Omega} \nabla y(x) \nabla T_{k}(z)(x) d x-\int_{\Omega} u(x) T_{k}(z)(x) d x\right\} \\
& =\int_{\Omega} \nabla y(x) \nabla z(x) d x-\int_{\Omega} u(x) z(x) d x<+\infty .
\end{aligned}
$$

Next, for a general $z \in H_{0}^{1}(\Omega)$, we notice that $z=z^{+}-z^{-}$with $z^{+}, z^{-} \in$ $H_{0}^{1}(\Omega)$. This proves that $(2.1)$ is satisfied. Moreover $\mathrm{e}^{y} \in H^{-1}(\Omega)$ and $(2.1)$ shows that for all $z \in H_{0}^{1}(\Omega)$

$$
\left\langle\mathrm{e}^{y}, z\right\rangle=\int_{\Omega} \mathrm{e}^{y(x)} z(x) d x .
$$

Now we state a very important identity to derive some estimates, in terms of $u$, on the solution of (1.1).

THEOREM 2.2. Let $u \in L^{2}(\Omega)$ and assume that $y \in H^{2}(\Omega)$ is a solution of (1.1). Then for any $x_{0} \in \mathbb{R}^{n}$ we have

$$
\begin{gathered}
\left(\frac{n}{2}-1\right) \int_{\Omega}|\nabla y(x)|^{2} d x+\frac{1}{2} \int_{\Gamma}|\nabla y(\sigma)|^{2}\left[\nu(\sigma) \cdot\left(\sigma-x_{0}\right)\right] d \sigma \\
=n \int_{\Omega}\left(\mathrm{e}^{y(x)}-1\right) d x-\int_{\Omega} u(x)\left[\left(x-x_{0}\right) \cdot \nabla y(x)\right] d x,
\end{gathered}
$$

where $\nu(\sigma)$ denotes the outward unit normal vector to $\Gamma$ at the point $\sigma$. 
Proof. Since $y \in H^{2}(\Omega)$, then $\mathrm{e}^{y}=-\Delta y-u \in L^{2}(\Omega)$. Therefore we can multiply the equation (1.1) by any function of $L^{2}(\Omega)$ and make the integration in $\Omega$. In particular we can take $\left(x-x_{0}\right) \cdot \nabla y(x) \in L^{2}(\Omega)$ as this function:

$$
\int_{\Omega}\left[-\Delta y\left[\left(x-x_{0}\right) \cdot \nabla y\right]=\int_{\Omega} \mathrm{e}^{y}\left[\left(x-x_{0}\right) \cdot \nabla y\right] d x+\int_{\Omega} u\left[\left(x-x_{0}\right) \cdot \nabla y\right] d x .\right.
$$

Let us make an integration by parts in the first integral

$$
\begin{aligned}
& \int_{\Omega}\left[-\Delta y\left[\left(x-x_{0}\right) \cdot \nabla y\right]\right. \\
= & \int_{\Omega} \nabla y \nabla\left[\left(x-x_{0}\right) \cdot \nabla y\right]-\int_{\Gamma} \partial_{\nu} y(\sigma)\left[\left(\sigma-x_{0}\right) \cdot \nabla y(\sigma)\right] d \sigma \\
= & \sum_{i, j=1}^{n} \int_{\Omega} \partial_{x_{i}} y \partial_{x_{i}}\left[\left(x_{j}-x_{0 j}\right) \partial_{x_{j}} y\right] d x-\int_{\Gamma} \partial_{\nu} y(\sigma)\left[\left(\sigma-x_{0}\right) \cdot \nabla y(\sigma)\right] d \sigma \\
= & \left.\sum_{i=1}^{n} \int_{\Omega}\left|\partial_{x_{i}} y\right|^{2} d x+\sum_{i, j=1}^{n} \int_{\Omega} \partial_{x_{i}} y\left(x_{j}-x_{0 j}\right) \partial_{x_{i} x_{j}}^{2} y\right] d x \\
- & \int_{\Gamma} \partial_{\nu} y(\sigma)\left[\left(\sigma-x_{0}\right) \cdot \nabla y(\sigma)\right] d \sigma \\
= & \sum_{i=1}^{n} \int_{\Omega}\left|\partial_{x_{i}} y\right|^{2} d x+\frac{1}{2} \sum_{i, j=1}^{n} \int_{\Omega}\left(x_{j}-x_{0 j}\right) \partial_{x_{j}}\left(\partial_{x_{i}} y\right)^{2} d x \\
- & \int_{\Gamma} \partial_{\nu} y(\sigma)\left[\left(\sigma-x_{0}\right) \cdot \nabla y(\sigma)\right] d \sigma .
\end{aligned}
$$

Now we can integrate by parts in the last relation, taking into account that for $\sigma \in \Gamma$ we have $\nabla y(\sigma)= \pm|\nabla y(\sigma)| \nu(\sigma)$, then $\left(\sigma-x_{0}\right) \cdot \nabla y(\sigma)=$ $\pm|\nabla y(\sigma)| \nu(\sigma) \cdot\left(\sigma-x_{0}\right)$ and $\partial_{\nu} y(\sigma)=\nabla y(\sigma) \cdot \nu(\sigma)= \pm|\nabla y(\sigma)|$, we get

$$
\begin{aligned}
& \int_{\Omega}\left[-\Delta y\left[\left(x-x_{0}\right) \cdot \nabla y\right]=\sum_{i=1}^{n} \int_{\Omega}\left|\partial_{x_{i}} y\right|^{2} d x\right. \\
& +\frac{1}{2} \sum_{i, j=1}^{n}\left\{-\int_{\Omega}\left(\partial_{x_{i}} y\right)^{2}+\int_{\Gamma}\left(x_{j}-x_{0 j}\right) \nu_{j}(x)\left(\partial_{x_{i}} y\right)^{2} d \sigma\right\} \\
& -\int_{\Gamma} \partial_{\nu} y(\sigma)\left[\left(\sigma-x_{0}\right) \cdot \nabla y(\sigma)\right] d \sigma \\
& =\left(1-\frac{n}{2}\right) \sum_{i=1}^{n} \int_{\Omega}|\nabla y|^{2} d x-\frac{1}{2} \int_{\Gamma}\left[\nu(\sigma) \cdot\left(\sigma-x_{0}\right)\right]|\nabla y(\sigma)|^{2} d \sigma .
\end{aligned}
$$

In order to handle the first term in the right hand side of (2.4), for $k \geq 1$ set

$$
T_{k}(y)(x):= \begin{cases}+k & \text { if } y(x)>+k \\ y(x) & \text { if }-k \leq y(x) \leq k \\ -k & \text { if } y(x)<-k\end{cases}
$$


One checks easily that $T_{k}(y) \rightarrow y$ in $H_{0}^{1}(\Omega)$ and a.e., $\mathrm{e}^{T_{k}(y)} \rightarrow \mathrm{e}^{y}$ in $L^{2}(\Omega)$ and a.e. On the other hand $\mathrm{e}^{T_{k}(y)} \nabla T_{k}(y) \rightarrow \mathrm{e}^{y} \nabla y$ in $L^{1}(\Omega)$; but as $\nabla\left(\mathrm{e}^{T_{k}(y)}\right)=$ $\mathrm{e}^{T_{k}(y)} \nabla T_{k}(y)$, we conclude that $\mathrm{e}^{y} \nabla y=\nabla\left(\mathrm{e}^{y}-1\right)$ in $L^{1}(\Omega)$. Therefore

$$
\int_{\Omega} \mathrm{e}^{y} \nabla y \cdot \Phi d x=-\int_{\Omega}\left(\mathrm{e}^{y}-1\right) \nabla \cdot \Phi d x
$$

for all $\Phi \in\left(C^{1}\left(\mathbb{R}^{n}\right)\right)^{n}$, and we may write:

$$
\begin{gathered}
\int_{\Omega} \mathrm{e}^{y}\left[\left(x-x_{0}\right) \cdot \nabla y\right] d x=\sum_{j=1}^{n} \int_{\Omega}\left(x_{j}-x_{0 j}\right) \mathrm{e}^{y} \partial_{x_{j}} y d x \\
=\sum_{j=1}^{n} \int_{\Omega}\left(x_{j}-x_{0 j}\right) \partial_{x_{j}}\left[\mathrm{e}^{y}-1\right] d x-\sum_{j=1}^{n} \int_{\Omega}\left[\mathrm{e}^{y}-1\right] d x=-n \int_{\Omega}\left[\mathrm{e}^{y}-1\right] d x .
\end{gathered}
$$

Putting together (2.5) and (2.6) in (2.4), we get (2.3).

LEMMA 2.3. Let us assume that $u \in L^{2}(\Omega), \Omega$ is star-shaped with respect to some interior point $x_{0}$ (i.e. $\left(\sigma-x_{0}\right) \cdot \nu(\sigma) \geq 0$ for $\left.\sigma \in \Gamma\right)$ and $y \in H_{0}^{1}(\Omega)$ satisfies $\Delta y \in L^{2}(\Omega)$. Then we have

$$
\left(\frac{n}{2}-1\right) \int_{\Omega}|\nabla y(x)|^{2} d x \leq \int_{\Omega} \Delta y\left[\left(x-x_{0}\right) \cdot \nabla y\right] d x
$$

Proof. Let us consider a sequence of bounded open sets $\Omega_{1} \supset \Omega_{2} \supset \cdots \supset \bar{\Omega}$, with $\cap_{j=1}^{\infty} \Omega_{j}=\bar{\Omega}$, with $\Gamma_{j}=\partial \Omega_{j}$ of class $C^{1,1}$ and such that all of them are star-shaped with respect to $x_{0}$. For each $j$ we consider the problem

$$
\begin{cases}-\Delta z=\left(\mathrm{e}^{y}+u\right) \chi_{\Omega} & \text { in } \Omega_{j} \\ z=0 & \text { on } \Gamma_{j},\end{cases}
$$

where $\chi_{\Omega}$ is the characteristic function of $\Omega$. This linear problem has a unique solution $y_{j} \in H_{0}^{1}\left(\Omega_{j}\right) \cap H^{2}\left(\Omega_{j}\right)$. We extend each $y_{j}$ and $y$ to $\Omega_{1}$ by zero, thus $y_{j}, y \in H_{0}^{1}\left(\Omega_{1}\right)$. From the above equation we get

$$
\int_{\Omega_{1}}\left|\nabla y_{j}\right|^{2} d x=\int_{\Omega_{j}}\left|\nabla y_{j}\right|^{2} d x=\int_{\Omega}\left[\mathrm{e}^{y}+u\right] y_{j} d x \leq\left\|\mathrm{e}^{y}+u\right\|_{L^{2}(\Omega)}\left\|y_{j}\right\|_{L^{2}(\Omega)},
$$

which proves the boundedness of $\left\{y_{j}\right\}_{j=1}^{\infty}$ in $H_{0}^{1}\left(\Omega_{1}\right)$. By taking a subsequence we can assume that $y_{j} \rightarrow \tilde{y}$ weakly in $H_{0}^{1}\left(\Omega_{1}\right)$ and $y_{j}(x) \rightarrow \tilde{y}(x)$ for almost all $x \in \Omega_{1}$. Obviously we have that $\tilde{y}(x)=0$ for $x \in \Omega_{1} \backslash \bar{\Omega}$. Then we have that $\tilde{y}=0$ on $\Gamma$ and $-\Delta \tilde{y}=\lim _{j \rightarrow \infty}-\Delta y_{j}=\mathrm{e}^{y}+u$ in $\Omega$. This leads to the equality $\tilde{y}=y$. Moreover

$$
\begin{aligned}
\int_{\Omega_{1}}|\nabla y|^{2} d x & \leq \liminf _{j \rightarrow \infty} \int_{\Omega_{1}}\left|\nabla y_{j}\right|^{2} d x \leq \limsup _{j \rightarrow \infty} \int_{\Omega_{1}}\left|\nabla y_{j}\right|^{2} d x \\
& =\limsup _{j \rightarrow \infty} \int_{\Omega_{j}}\left|\nabla y_{j}\right|^{2} d x=\lim _{j \rightarrow \infty} \int_{\Omega_{j}}\left[\mathrm{e}^{y}+u\right] y_{j} d x \\
& =\int_{\Omega}\left[\mathrm{e}^{y}+u\right] y d x=\int_{\Omega}|\nabla y|^{2} d x=\int_{\Omega_{1}}|\nabla y|^{2} d x,
\end{aligned}
$$

hence $\lim _{j \rightarrow \infty}\left\|y_{j}\right\|_{H_{0}^{1}\left(\Omega_{1}\right)}=\|y\|_{H_{0}^{1}\left(\Omega_{1}\right)}$, which proves the strong convergence $y_{j} \rightarrow y$ in $H_{0}^{1}\left(\Omega_{1}\right)$. 
Now arguing again as in the proof of Theorem 2.2 and using the fact that $\left(\sigma-x_{0}\right) \cdot \nu_{j}(\sigma) \geq 0$ for every $\sigma \in \Gamma_{j}$ (here $\nu_{j}$ is the outward normal to $\Gamma_{j}$ ), we deduce

$$
\begin{aligned}
& \int_{\Omega}(-\Delta y)\left[\left(x-x_{0}\right) \cdot \nabla y\right] d x=\lim _{j \rightarrow \infty} \int_{\Omega}\left(-\Delta y_{j}\right)\left[\left(x-x_{0}\right) \cdot \nabla y_{j}\right] d x \\
& =\lim _{j \rightarrow \infty} \int_{\Omega_{j}}\left(-\Delta y_{j}\right)\left[\left(x-x_{0}\right) \cdot \nabla y_{j}\right] d x \\
& =\lim _{j \rightarrow \infty}\left\{\left(1-\frac{n}{2}\right) \int_{\Omega_{j}}\left|\nabla y_{j}\right|^{2} d x-\frac{1}{2} \int_{\Gamma_{j}}\left[\left(\sigma-x_{0}\right) \cdot \nu_{j}(\sigma)\right]\left|\nabla y_{j}\right|^{2} d \sigma\right\} \\
& \leq \lim _{j \rightarrow \infty}\left(1-\frac{n}{2}\right) \int_{\Omega}\left|\nabla y_{j}\right|^{2} d x=\left(1-\frac{n}{2}\right) \int_{\Omega}|\nabla y|^{2} d x .
\end{aligned}
$$

Remark. Inequality (2.7) makes sense when we assume only that $y \in Y$ and $u \in L^{2}(\Omega)$ but we do not know whether it holds under these assumptions. Actually (2.7) is true for solutions $(y, u) \in Y \times K$ such that there is a sequence of solutions $\left(y_{k}, u_{k}\right) \in Y \times K$ with $y_{k} \in H^{2}(\Omega)$ satisfying (1.1), $u_{k} \rightarrow u$ strongly in $L^{2}(\Omega)$ (indeed this implies that $y_{k} \rightarrow y$ in $H_{0}^{1}(\Omega)$-weak and $\mathrm{e}^{y_{k}} \rightarrow \mathrm{e}^{y}$ in $\left.L^{1}(\Omega)\right)$.

Corollary 2.4. Let us assume that $u \in L^{2}(\Omega), \Omega$ is star-shaped with respect to some interior point $x_{0}$ and $y \in Y$ is a solution of (1.1) such that $\mathrm{e}^{y} \in L^{2}(\Omega)$. Then we have

$$
\left(\frac{n}{2}-1\right) \int_{\Omega}|\nabla y(x)|^{2} d x \leq n \int_{\Omega}\left(\mathrm{e}^{y(x)}-1\right) d x-\int_{\Omega} u(x)\left[\left(x-x_{0}\right) \cdot \nabla y(x)\right] d x .
$$

Proof. If $y \in H^{2}(\Omega)$ this inequality is an immediate consequence of $(2.3)$. Indeed it is enough to note that $\nu(\sigma) \cdot\left(\sigma-x_{0}\right) \geq 0$ for almost every $\sigma \in \Gamma$ because $\Omega$ is assumed to be star-shaped with respect to $x_{0}$. Since we have not assumed $\Gamma$ to be of class $C^{1,1}$ or $\Omega$ to be convex, we cannot deduce the $H^{2}(\Omega)$-regularity of $y$ from the fact that $\mathrm{e}^{y}+u \in L^{2}(\Omega)$. However (2.6) is still valid, therefore the result follows from the previous lemma.

As a consequence of this corollary, we deduce some estimates for $y$ in terms of $u$.

THEOREM 2.5. Let us assume that $y$ is a solution of (1.1) that satisfies the inequality (2.8). Then there exist positive constants $C_{i}, 1 \leq i \leq 4$, independent of $u$ and $y$ such that

$$
\begin{gathered}
\left\|\mathrm{e}^{y} y\right\|_{L^{1}(\Omega)} \leq C_{1}\|u\|_{L^{2}(\Omega)}^{2}+C_{2}, \\
\|y\|_{H_{0}^{1}(\Omega)} \leq C_{3}\|u\|_{L^{2}(\Omega)}+C_{4} .
\end{gathered}
$$

Proof. Thanks to Theorem 2.1 we know that

$$
\int_{\Omega}|\nabla y(x)|^{2} d x=\int_{\Omega} \mathrm{e}^{y(x)} y(x) d x+\int_{\Omega} u(x) y(x) d x .
$$


Combining this identity with $(2.8)$ we deduce

$$
\begin{aligned}
& \left(\frac{n}{2}-1\right) \int_{\Omega} \mathrm{e}^{y} y d x+\left(\frac{n}{2}-1\right) \int_{\Omega} u y d x \\
& \leq n \int_{\Omega}\left[\mathrm{e}^{y}-1\right] d x-\int_{\Omega} u\left[\left(x-x_{0}\right] \cdot \nabla y\right] d x .
\end{aligned}
$$

Which yields

$$
\int_{\Omega} \mathrm{e}^{y} y d x \leq \frac{2 n}{n-2} \int_{\Omega}\left[\mathrm{e}^{y}-1\right] d x+c_{1}\|u\|_{L^{2}(\Omega)}\|y\|_{H_{0}^{1}(\Omega)} .
$$

Let 11 set

$$
\Omega_{n}=\left\{x \in \Omega: y(x)>\frac{4 n}{n-2}\right\} .
$$

Then from (2.12) we get

$$
\int_{\Omega} \mathrm{e}^{y} y d x \leq \frac{1}{2} \int_{\Omega_{n}} \mathrm{e}^{y} y d x+\int_{\Omega \backslash \Omega_{n}} \mathrm{e}^{\frac{4 n}{n-2}} d x+c_{1}\|u\|_{L^{2}(\Omega)}\|y\|_{H_{0}^{1}(\Omega)},
$$

therefore

$$
\frac{1}{2} \int_{\Omega} \mathrm{e}^{y} y d x \leq c_{2}-\frac{1}{2} \int_{\Omega \backslash \Omega_{n}} \mathrm{e}^{y} y d x+c_{1}\|u\|_{L^{2}(\Omega)}\|y\|_{H_{0}^{1}(\Omega)} .
$$

Taking into account that $\mathrm{e}^{t}|t| \leq c(n)<+\infty$ for any real number $t \leq \frac{4 n}{n-2}$, we get from the previous inequality

$$
\int_{\Omega} \mathrm{e}^{y}|y| d x \leq c_{3}+c_{1}\|u\|_{L^{2}(\Omega)}\|y\|_{H_{0}^{1}(\Omega)} .
$$

Using this inequality in (2.11), we obtain (2.10). Finally using (2.10) in (2.13) we get the estimate (2.9).

We finish this section by proving two propositions that will be very useful in the next sections. Here we will use the notation $y^{+}=\max \{y, 0\}$ and $y^{-}=\max \{-y, 0\}$.

Proposition 2.6. Let $y$ be a solution of (1.1) corresponding to a function $u \in L^{p}(\Omega)$. Then $y^{+} \in L^{r}(\Omega)$ for all $1 \leq r<+\infty ; y^{-} \in L^{q}(\Omega)$ with

$$
q=\left\{\begin{array}{cl}
+\infty & \text { if } p>n / 2, \\
<+\infty & \text { if } p=n / 2, \\
n p /(n-2 p) & \text { if } p<n / 2 .
\end{array}\right.
$$

Moreover $\left\|y^{-}\right\|_{L^{q}(\Omega)} \leq C_{p, q}\left\|u^{-}\right\|_{L^{p}(\Omega)}$ for some constant $C_{p, q}>0$ independent of $u$ and $y$; if we denote by $[r]$ the integer part of $r$ and $k:=[r]+1$ then

$$
\left\|y^{+}\right\|_{L^{r}(\Omega)} \leq(k !)^{1 / r}\left(C_{1}\|u\|_{L^{2}(\Omega)}+C_{2}\right)^{1 / r} .
$$

Proof. Let us take $k=[r]+1$. Then $\left|y^{+}\right|^{r}<k ! \mathrm{e}^{y} \in L^{1}(\Omega)$, which proves that $y^{+} \in L^{r}(\Omega)$. Using the estimate $\int_{\Omega} \mathrm{e}^{y} d x \leq C_{1}\|u\|_{L^{2}(\Omega)}^{2}+C_{2}$ one gets the last estimate of the proposition.

Now assume that $p<n / 2$ (the case $p \geq n / 2$ may be treated in the same way). Consider $\psi \in H_{0}^{1}(\Omega)$ satisfying $-\Delta \psi=-u^{-}$. We have

$$
-\Delta y \geq-u^{-}=-\Delta \psi
$$


and by the maximum principle we conclude that $y \geq \psi$ in $\Omega$. As $\psi<0$, we conclude that $0 \leq y^{-} \leq \psi^{-}=|\psi|$. Now recall that by a classical result of G. Stampacchia [12] (theorems 4.1 and 4.2), if for some $s \geq 2$ (and $s<n$ ) one has $-\Delta z=T \in W^{-1, s}(\Omega)$ and $z \in H_{0}^{1}(\Omega)$, then

$$
\|z\|_{L^{s^{*}}(\Omega)} \leq C(n, s)\left(\|T\|_{W-1, s(\Omega)}+\|z\|_{L^{2}(\Omega)}\right), \quad \frac{1}{s^{*}}=\frac{1}{s}-\frac{1}{n}
$$

Here we have $-\Delta \psi=-u^{-} \in L^{p}(\Omega)$ and $L^{p}(\Omega) \subset W^{-1, s}(\Omega)$ if

$$
\frac{1}{s}=\frac{1}{p}-\frac{1}{n}
$$

Consequently one sees that the estimate of the proposition on $\left\|y^{-}\right\|_{L^{q}(\Omega)}$ holds if $q=s^{*}$, which means

$$
\frac{1}{q}=\frac{1}{p}-\frac{2}{n}
$$

Proposition 2.7. Let $u$ and $y$ be as in Proposition 2.6 and satisfying the inequality (2.8). Then $y \in L^{p}(\Omega)$ and

$$
\|y\|_{L^{p}(\Omega)} \leq C_{1, p}\|u\|_{L^{p}(\Omega)}+C_{2, p},
$$

for some constants $C_{i, p}>0$ independent of $u$ and $y$.

Proof. Let us take $k=[p]+1$. From (2.9), the inequality $\mathrm{e}^{t} \leq|t| \mathrm{e}^{t}+1$ for every $t \in \mathbb{R}$ and taking into account that $p \geq 2$, we deduce

$$
\begin{gathered}
\left\|y^{+}\right\|_{L^{p}(\Omega)} \leq\left(k ! \int_{\Omega} \mathrm{e}^{y} d x\right)^{\bar{p}} \leq\left(c_{1}\|u\|_{L^{2}(\Omega)}^{2}+c_{2}\right)^{1 / p} \\
\leq c_{3}\|u\|_{L^{2}(\Omega)}^{2 / p}+c_{4} \leq c_{3}\left(\|u\|_{L^{2}(\Omega)}+1\right)+c_{4} \leq c_{5}\|u\|_{L^{p}(\Omega)}+c_{6} .
\end{gathered}
$$

On the other hand, from Proposition 2.6 it follows that $y^{-} \in L^{p}(\Omega)$. Indeed it is enough to note that $n p /(n-2 p)>p$ if $p<n / 2$. Moreover

$$
\left\|y^{-}\right\|_{L^{p}(\Omega)} \leq C_{p}\left\|u^{-}\right\|_{L^{p}(\Omega)} \cdot
$$

Now it is enough to write $y=y^{+}-y^{-}$and to use the two inequalities obtained above to achieve the desired result.

\section{Existence of an Optimal Control. Case $p>2$}

The aim of this section is to study the existence of a solution for the optimal control problem. As usual, to prove the existence of such a solution, we take a minimizing sequence $\left\{\left(y_{k}, u_{k}\right)\right\}_{k=1}^{\infty}$ of feasible elements. Assuming that either $K$ is bounded in $L^{p}(\Omega)$ or $N>0$, we can deduce that $\left\{u_{k}\right\}_{k=1}^{\infty}$ is bounded. The difficult part is to deduce that $\left\{y_{k}\right\}_{k=1}^{\infty}$ is bounded in $H_{0}^{1}(\Omega)$. If the elements $\left(y_{k}, u_{k}\right)$ satisfy the inequality $(2.8)$, then Theorem 2.5 provides the necessary inequalities to deduce the bounded ness of $\left\{y_{k}\right\}_{k=1}^{\infty}$. Unfortunately (2.8) has been proved to hold only for solutions of the state equation with $\mathrm{e}^{y} \in L^{2}(\Omega)$. This leads us to consider the following class of states: we denote by $\mathcal{Y}$ the subset of $Y$ formed by the functions which satisfy (1.1) and (2.8) for some control $u$ (note that for $q>n / 2$ one has ESAIM: CoCv, November 1998, Vol. 3, 361-380 
$\left.W^{2, q}(\Omega) \cap H_{0}^{1}(\Omega) \subset \mathcal{Y}\right)$. Not every element of $\mathcal{Y}$ needs to be a solution of (1.1) with $\mathrm{e}^{y} \in L^{2}(\Omega)$. In fact we have the following result.

TheOREM 3.1. Let us assume that $\left\{\left(y_{k}, u_{k}\right)\right\}_{k=1}^{\infty} \subset \mathcal{Y} \times L^{p}(\Omega)$ is a sequence of functions satisfying (1.1) and converging weakly to some element $(y, u)$ in $H_{0}^{1}(\Omega) \times L^{p}(\Omega)$, with $p>2$. Then $\mathrm{e}^{y_{k}} \rightarrow \mathrm{e}^{y}$ in $L^{1}(\Omega), y \in \mathcal{Y}$ and $(y, u)$ satisfies also (1.1). The same result holds if $y_{k} \rightarrow y$ in $H_{0}^{1}(\Omega)$ and $u_{k} \rightarrow u$ strongly in $L^{2}(\Omega)$.

Proof. The weak convergence $y_{k} \rightarrow y$ in $H_{0}^{1}(\Omega)$ implies the strong convergence $y_{k} \rightarrow y$ in $L^{2}(\Omega)$. Then taking a subsequence if necessary, we can assume that $y_{k}(x) \rightarrow y(x)$ for almost every point $x \in \Omega$. In particular $\mathrm{e}^{y_{k}(x)} \rightarrow \mathrm{e}^{y(x)}$ almost everywhere in $\Omega$. Let us use Vitali's theorem to prove the convergence $\mathrm{e}^{y_{k}} \rightarrow \mathrm{e}^{y}$ in $L^{1}(\Omega)$. First let us note that the boundedness of $\left\{u_{k}\right\}_{k=1}^{\infty}$ in $L^{2}(\Omega)$ along with $(2.9)$ imply that $\left\|\mathrm{e}^{y_{k}} y_{k}\right\|_{L^{1}(\Omega)} \leq C$ for some constant $C<+\infty$ and all $k \in \mathbb{N}$. Now given $\epsilon>0$, let us take $m>0$ such that $C / m<\epsilon / 2$ and $\delta=\epsilon /\left(2 \mathrm{e}^{m}\right)$. Then for every measurable set $E \subset \Omega$, with meas $(E)<\delta$, we have

$$
\begin{gathered}
\int_{E} \mathrm{e}^{y_{k}(x)} d x \leq \frac{1}{m} \int_{\left\{x \in E: y_{k}(x)>m\right\}} \mathrm{e}^{y_{k}(x)} y_{k}(x) d x+\int_{\left\{x \in E: y_{k}(x) \leq m\right\}} \mathrm{e}^{m} d x \\
\leq \frac{1}{m} \int_{\Omega} \mathrm{e}^{y_{k}(x)}\left|y_{k}(x)\right| d x+\mathrm{e}^{m} \operatorname{meas}(E) \leq \frac{C}{m}+\mathrm{e}^{m} \delta<\epsilon \quad \forall k \in \mathbb{N},
\end{gathered}
$$

which allows to conclude the desired convergence. Now it is easy to pass to the limit in the state equation satisfied by $\left(y_{k}, u_{k}\right)$ and to conclude that $(u, y)$ satisfies (1.1).

Let us prove that $(y, u)$ satisfies (2.8). First of all we will prove that there exists a subsequence, that we will denote in the same way, such that $\nabla y_{k}(x) \rightarrow \nabla y(x)$ for almost all point $x \in \Omega$. To achieve this aim, we remark that $\mathrm{e}^{y_{k}} \rightarrow \mathrm{e}^{y}$ in $L^{1}(\Omega)$ while $\left(u_{k}\right)_{k}$ is bounded in $L^{p}(\Omega)$ : therefore $\left(\Delta y_{k}\right)_{k}$ is bounded in $L^{1}(\Omega)$. Now by a result due to L. Boccardo and F. Murat [2] (theorem 2.1) one may conclude that there exists a subsequence (still denoted by) $\left(y_{k}\right)_{k}$ such that $y_{k} \rightarrow y$ in $H^{1}(\Omega)$ weakly and $\nabla y_{k} \rightarrow \nabla y$ almost everywhere.

Now the weak convergence $\nabla y_{k} \rightarrow \nabla y$ in $\left(L^{2}(\Omega)\right)^{n}$ along with the pointwise convergence implies the strong convergence in $\left(L^{r}(\Omega)\right)^{n}$ for all $r<2$ (we use here the fact that the weak convergence in $L^{2}(\Omega)$ implies that the sequence $\left|\nabla y_{k}-\nabla y\right|^{r}$ is equi-integrable and we apply Vitali's theorem). In particular the strong convergence of $\nabla y_{k} \rightarrow \nabla y$ holds in $L^{p^{\prime}}(\Omega)$, with $(1 / p)+\left(1 / p^{\prime}\right)=1$ (this is the only place where we need the assumption $p>2$ ). Then we can pass to the limit in the inequality (2.8) to obtain:

$$
\begin{aligned}
\left(\frac{n}{2}-1\right) \int_{\Omega}|\nabla y|^{2} d x & \leq \liminf _{k \rightarrow \infty}\left(\frac{n}{2}-1\right) \int_{\Omega}\left|\nabla y_{k}\right|^{2} d x \\
& \leq \liminf _{k \rightarrow \infty}\left\{n \int_{\Omega}\left[\mathrm{e}^{y_{k}}-1\right] d x-\int_{\Omega} u_{k}\left[\left(x-x_{0}\right) \cdot \nabla y_{k} d x\right\}\right. \\
& =n \int_{\Omega}\left[\mathrm{e}^{y}-1\right] d x-\int_{\Omega} u\left[\left(x-x_{0}\right) \cdot \nabla y d x,\right.
\end{aligned}
$$


which concludes the proof when $\left(y_{k}, u_{k}\right) \rightarrow(y, u)$ in $H_{0}^{1}(\Omega) \times L^{p}(\Omega)$ and $p>2$. On the other hand it is clear that when $p=2$ and $u_{k} \rightarrow u$ strongly in $L^{2}(\Omega)$, in the above inequalities one has

$$
\int_{\Omega} u_{k}\left(x-x_{0}\right) \cdot \nabla y_{k} d x \rightarrow \int_{\Omega} u\left(x-x_{0}\right) \cdot \nabla y d x
$$

as $k \rightarrow \infty$.

Now we reformulate the control problem as follows

$$
(\mathcal{P})\left\{\begin{array}{l}
\text { Minimize } J(y, u):=\int_{\Omega} L(x, y(x)) d x+\frac{N}{p} \int_{\Omega}|u(x)|^{p} d x \\
(y, u) \in \mathcal{Y} \times K \text { satisfies (1.1). }
\end{array}\right.
$$

The fact of taking $(y, u) \in \mathcal{Y} \times K$ imposes a restriction on the class of solutions of (1.1), but it is not restrictive with respect to the controls. More precisely, we have the following result

PRoposition 3.2. Let us assume that $\Omega$ is star-shaped with respect to some point $x_{0} \in \Omega$ and that (1.1) has a solution for some control $u \in L^{p}(\Omega)$, $p \geq 2$. Then there exists a solution $z$ of (1.1) corresponding to the same control $u$ and belonging to the class $\mathcal{Y}$.

Before proving this proposition, we state and prove the following lemma:

Lemma 3.3. For $k \geq 1$ and $t \in \mathbb{R}$ let us denote $f_{k}(t):=\min \left\{\mathrm{e}^{k}, \mathrm{e}^{t}\right\}$. Then there is $z_{k} \in H_{0}^{1}(\Omega)$ such that

$$
\begin{cases}-\Delta z=f_{k}(z)+u & \text { in } \Omega, \\ z_{k}=0 & \text { on } \Gamma .\end{cases}
$$

Moreover $z_{k} \leq z_{k+1}$ and the sequence $\left\{z_{k}\right\}_{k=1}^{\infty}$ is bounded in $H_{0}^{1}(\Omega)$.

Proof. We denote with $y$ a solution of (1.1) associated to the control $u$. Let us take $y_{0} \in H_{0}^{1}(\Omega)$ such that $-\Delta y_{0}=u$. Then we have the following three inequalities:

$$
-\Delta y_{0} \leq f_{k}\left(y_{0}\right)+u, \quad-\Delta y \geq f_{k}(y)+u, \quad-\Delta\left(y-y_{0}\right)=\mathrm{e}^{y}>0 .
$$

From the last relation we deduce that $y_{0} \leq y$ in $\Omega$. From the two first relations it follows that $y_{0}$ is a subsolution and $y$ is a supersolution of (3.1). Combining all these, by the now classical techniques introduced by D.H. Sattinger [10] (see also H. Amann [1]) we get the existence of a solution $z_{k}$ of (3.1) such that $y_{0} \leq z_{k} \leq y$. Moreover we have $z_{k} \leq z_{k+1}$ and

$$
\begin{aligned}
\int_{\Omega}\left|\nabla z_{k}\right|^{2} d x & =\int_{\Omega}\left[f_{k}\left(z_{k}\right)+u\right] z_{k} d x \leq \int_{\Omega}\left[\mathrm{e}^{z_{k}}+|u|\right]\left|z_{k}\right| d x \\
& \leq \int_{\Omega}\left[\mathrm{e}^{y}+|u|\right]\left|z_{k}\right| d x \leq\left\|\mathrm{e}^{y}+|u|\right\|_{H^{-1}(\Omega)}|| z_{k} \|_{H_{0}^{1}(\Omega)},
\end{aligned}
$$

which implies that $\left\{z_{k}\right\}_{k=1}^{\infty}$ is bounded in $H_{0}^{1}(\Omega)$.

Proof of Proposition 3.2. The sequence $\left\{z_{k}\right\}_{k=1}^{\infty}$ being given by Lemma 3.3, taking a subsequence, denoted in the same way, we infer that there exists an element $z \in H_{0}^{1}(\Omega)$ such that

$$
z_{k} \rightarrow z \text { weakly in } H_{0}^{1}(\Omega), \quad z_{k}(x) \rightarrow z(x) \text { a.e. } x \in \Omega \text {. }
$$


Therefore $f_{k}\left(z_{k}(x)\right) \rightarrow \mathrm{e}^{z(x)}$ for almost all $x \in \Omega$ and $f_{k}\left(z_{k}\right) \leq \mathrm{e}^{z_{k}} \leq \mathrm{e}^{y}$. Then we can apply the dominated convergence theorem to obtain that $\mathrm{e}^{z_{k}} \rightarrow$ $\mathrm{e}^{z}$ in $L^{1}(\Omega)$. Now it is easy to pass to the limit and to deduce that $z$ satisfies (1.1). We are going to prove that $z \in \mathcal{Y}$. First of all let us remark that $f_{k}\left(z_{k}\right)+u \in L^{2}(\Omega)$, hence $\Delta z_{k} \in L^{2}(\Omega)$. Therefore we can multiply (3.1) by $\left(x-x_{0}\right) \cdot \nabla z_{k}$ and integrate over $\Omega$ and get:

$$
\begin{gathered}
-\int_{\Omega} \Delta z_{k}\left[\left(x-x_{0}\right) \cdot \nabla z_{k}\right] d x \\
=\int_{\Omega} f_{k}\left(z_{k}\right)\left[\left(x-x_{0}\right) \cdot \nabla z_{k}\right] d x+\int_{\Omega} u\left[\left(x-x_{0}\right) \cdot \nabla z_{k}\right] d x .
\end{gathered}
$$

Let us define the function $F_{k}: \mathbb{R} \longrightarrow \mathbb{R}$ by

$$
F_{k}(t)=\left\{\begin{array}{cl}
\mathrm{e}^{t}-1 & \text { if } t \leq k, \\
\mathrm{e}^{k}(t-k+1)-1 & \text { if } t>k .
\end{array}\right.
$$

Then $F_{k}$ is the primitive of $f_{k}$ (which is defined in Lemma 3.3) satisfying $F_{k}(0)=0$ and $F_{k}(t) \leq \mathrm{e}^{t}-1$. Arguing as in the proof of Theorem 2.2 we obtain

$$
\int_{\Omega} f_{k}\left(z_{k}\right)\left[\left(x-x_{0}\right) \cdot \nabla z_{k}\right] d x=-n \int_{\Omega} F_{k}\left(z_{k}\right) d x \geq-n \int_{\Omega}\left[\mathrm{e}^{z_{k}}-1\right] d x
$$

Now from Lemma 2.3 we get

$$
\left(\frac{n}{2}-1\right) \int_{\Omega}\left|\nabla z_{k}\right|^{2} d x \leq \int_{\Omega}\left(\Delta z_{k}\right)\left[\left(x-x_{0}\right) \cdot \nabla z_{k}\right] d x .
$$

Combining (3.3), (3.4) and (3.5), the following inequality is obtained

$$
\left(\frac{n}{2}-1\right) \int_{\Omega}\left|\nabla z_{k}\right|^{2} d x \leq n \int_{\Omega}\left[\mathrm{e}^{z_{k}}-1\right] d x-\int_{\Omega} u\left[\left(x-x_{0}\right) \cdot \nabla z_{k}\right] d x .
$$

Using (3.2) and the convergence $\mathrm{e}^{z_{k}} \rightarrow \mathrm{e}^{z}$ in $L^{1}(\Omega)$, it comes

$$
\left(\frac{n}{2}-1\right) \int_{\Omega}|\nabla z|^{2} d x \leq n \int_{\Omega}\left[\mathrm{e}^{z}-1\right] d x-\int_{\Omega} u\left[\left(x-x_{0}\right) \cdot \nabla z\right] d x
$$

which proves that $z \in \mathcal{Y}$ as desired.

In the next proposition we state some interesting properties of the set of feasible controls.

Proposition 3.4. Let $p \geq 2$ and let $\Omega$ be star-shaped with respect to one of its interior points. Then the set of controls $u \in L^{p}(\Omega)$ for which there exists a solution $y \in \mathcal{Y}$ is non empty, convex and closed in $L^{p}(\Omega)$.

Proof. It is enough to take any function $y \in C_{c}^{\infty}(\Omega)$ and to put $u=-\Delta y-\mathrm{e}^{y}$ to deduce that the set of feasible controls is non empty. Let us take a sequence of feasible controls $\left\{u_{k}\right\}_{k=1}^{\infty}$ converging in $L^{p}(\Omega)$ to some function $u$. Thanks to Theorem 2.5 , we know that the corresponding states $\left\{y_{k}\right\}_{k=1}^{\infty} \subset \mathcal{Y}$ are bounded in $H_{0}^{1}(\Omega)$. Then Theorem 3.1 claims that any weak limit of $\left\{y_{k}\right\}_{k=1}^{\infty}$ is an element of $\mathcal{Y}$ satisfying (1.1) along with the control $u$, which proves that the set of feasible controls is closed.

Now let us prove the convexity. If $u_{1}$ and $u_{2}$ are two feasible controls, with associated states $y_{1}$ and $y_{2}$, respectively, and $\lambda \in(0,1)$, we set $u=$ 
$\lambda u_{1}+(1-\lambda) u_{2}$ and $y=\lambda y_{1}+(1-\lambda) y_{2}$. Let us take $\psi \in H_{0}^{1}(\Omega)$ such that $-\Delta \psi=u$. Then

$$
-\Delta \psi \leq \mathrm{e}^{\psi}+u, \text { and }-\Delta y=\lambda \mathrm{e}^{y_{1}}+(1-\lambda) \mathrm{e}^{y_{2}}+u \geq \mathrm{e}^{y}+u .
$$

Therefore $\psi$ is a subsolution of (1.1) for the control $u$ and $y$ is a supersolution. On the other hand, $-\Delta(y-\psi) \geq \mathrm{e}^{y}>0$, then $\psi \leq y$. Therefore we deduce the existence of a solution $z$ of (1.1) associated to the control $u$, with $\psi \leq z \leq y$. Finally, the conclusion follows from Proposition 3.2.

So far we have studied the properties of the feasible pairs $(y, u) \in \mathcal{Y} \times$ $L^{p}(\Omega)$ satisfying (1.1). Let us say something about the action of functional $J$ on these pairs. For each one of these pairs $(y, u), J$ is well defined and $-\infty<J(y, u) \leq+\infty$. Indeed the only trouble can come from the integral of $L(x, y(x))$. With the notation of (1.4), let us set

$$
f(x)=L(x, y(x))+\alpha_{2}\left(\left|y^{-}(x)\right|^{\theta_{2}}+\mathrm{e}^{y(x)}\right)-a_{2}(x) .
$$

Then $f$ is a nonnegative measurable function and consequently its integral is well defined as a number in $[0,+\infty]$. On the other hand, it is enough to use Theorem 2.5 and Proposition 2.6 and the assumptions on $\theta_{2}$ to deduce that $|y|^{\theta_{2}}$ and $\mathrm{e}^{y}$ are integrable functions. Therefore the integral of $L(x, y(x))$ is well defined, though it could be $+\infty$ in some cases.

Finally we establish our result of existence of a solution to $(\mathcal{P})$.

TheOrem 3.5. Let us assume that $p>2$ an

(i) There exists a pair $(y, u) \in \mathcal{Y} \times K$ satisfying (1.1).

(ii) Either $K$ is bounded or $N>0$.

Then problem $(\mathcal{P})$ has at least one solution.

Proof. Let us assume that $\mu:=\inf (\mathcal{P})<+\infty$ (otherwise the theorem is obvious). Let $\left\{\left(y_{k}, u_{k}\right)\right\}_{k=1}^{\infty}$ be a minimizing sequence for problem $(\mathcal{P})$ : $J\left(y_{k}, u_{k}\right) \downarrow \mu$. Thus we can suppose that $J\left(y_{k}, u_{k}\right) \leq \mu+1$. The main point in the proof is to establish the boundedness of $\left\{u_{k}\right\}_{k=1}^{\infty}$ in $L^{p}(\Omega)$. This is obvious if $K$ is bounded. Let us assume that $K$ is not bounded. We have

$$
\begin{aligned}
& \int_{\Omega} a_{2}(x) d x+\frac{N}{p} \int_{\Omega}\left|u_{k}(x)\right|^{p} d x-\alpha_{2} \int_{\Omega}\left|y_{k}^{-}(x)\right|^{\theta_{2}} d x-\alpha_{2} \int_{\Omega} \mathrm{e}^{y_{k}(x)} d x \\
& \leq J\left(y_{k}, u_{k}\right) \leq \mu+1 .
\end{aligned}
$$

Using the fact that the exponent $q$ defined in proposition 2.6 satisfies $q>p$ we conclude that $\left\|y_{k}^{-}\right\|_{\theta_{2}}^{\theta_{2}} \leq C\left\|u_{k}\right\|_{p}^{\theta_{2}}$. In the same way we observe that

$$
\int_{\Omega} \mathrm{e}^{y_{k}(x)} d x \leq C\left(\left\|u_{k}\right\|_{2}^{2}+1\right)
$$

and finally we obtain:

$$
\left\|u_{k}\right\|_{p}^{p} \leq C+C\left\|u_{k}\right\|_{p}^{2}+C\left\|u_{k}\right\|_{p}^{\theta_{2}} .
$$

As $\theta_{2}<p$ and $p>2$, this implies that $\left(u_{k}\right)_{k}$ is bounded in $L^{p}(\Omega)$.

The boundedness of $\left\{y_{k}\right\}_{k=1}^{\infty}$ in $H_{0}^{1}(\Omega)$ is a consequence of (2.10). Therefore, taking a subsequence if necessary, we can assume that $\left(y_{k}, u_{k}\right) \rightarrow(y, u)$ weakly in $H_{0}^{1}(\Omega) \times L^{p}(\Omega)$. Theorem 3.1 asserts that $y \in \mathcal{Y}$ and $(y, u)$ satisfies (1.1). Moreover the convexity and closedness of $K$ in $L^{p}(\Omega)$ implies that $u \in K$. Thus $(y, u)$ is a feasible pair for problem $(\mathcal{P})$. Let us prove that it ESAIM: COCV, NOVEMBer 1998, VoL. 3, 361-380 
is a solution. Since $y_{k} \rightarrow y$ strongly in $L^{2}(\Omega)$ we can take a subsequence in such a way that $y_{k}(x) \rightarrow y(x)$ for almost all $x \in \Omega$. Let us set

$$
f_{k}(x)=L\left(x, y_{k}(x)\right)+\alpha_{2}\left(\left|y_{k}^{-}(x)\right|^{\theta_{2}}+\mathrm{e}^{y_{k}(x)}\right)-a_{2}(x)
$$

and

$$
f(x)=L(x, y(x))+\alpha_{2}\left(|y(x)|^{\theta_{2}}+\mathrm{e}^{y(x)}\right)-a_{2}(x) .
$$

Then $f_{k}(x) \rightarrow f(x)$ almost everywhere and $f_{k} \geq 0$. Therefore we can apply Fatou's Lemma and the convergences $\mathrm{e}^{y_{k}} \rightarrow \mathrm{e}^{y}$ (Theorem 3.1) and $\left|y_{k}\right|^{\theta_{2}} \rightarrow|y|^{\theta_{2}}$ (Proposition 2.7) in $L^{1}(\Omega)$ to derive

$$
\begin{aligned}
J(y, u)= & \int_{\Omega} f(x) d x-\int_{\Omega}\left\{\alpha_{2}\left(\left|y^{-}(x)\right|^{\theta_{2}}+\mathrm{e}^{y(x)}\right)-a_{2}(x)\right\} d x \\
& +\frac{N}{p} \int_{\Omega}|u(x)|^{p} d x \leq \liminf _{k \rightarrow \infty}\left\{\int_{\Omega} f_{k}(x) d x\right. \\
& \left.-\int_{\Omega}\left[\alpha_{2}\left(\left|y_{k}^{-}(x)\right|^{\theta_{2}}+\mathrm{e}^{y_{k}(x)}\right)-a_{2}(x)\right] d x+\frac{N}{p} \int_{\Omega}\left|u_{k}(x)\right|^{p} d x\right\} \\
= & \liminf _{k \rightarrow \infty}\left\{\int_{\Omega} L\left(x, y_{k}(x)\right) d x+\frac{N}{p} \int_{\Omega}\left|u_{k}(x)\right|^{p} d x\right\} \\
= & \liminf _{k \rightarrow \infty} J\left(y_{k}, u_{k}\right)=\inf (\mathcal{P}) .
\end{aligned}
$$

This concludes the proof.

We conclude this section by studying the uniqueness of the solution.

THEOREM 3.6. Let $p \geq 2$ and assume that $\Omega$ is star-shaped with respect to some $x_{0} \in \Omega$ and that the set of admissible pairs $(y, u) \in \mathcal{Y} \times K$ satisfying (1.1) is not empty. Assume also that the function $t \mapsto L(x, t)$ is monotone on $\mathbb{R}$, non decreasing and convex for almost all $x \in \Omega$. Then problem $(\mathcal{P})$ has at most one solution if one of the following conditions holds:

(i) $N>0$;

(ii) $L(x, \cdot)$ is strictly increasing;

(iii) $L(x, \cdot)$ is strictly convex.

Proof. Let us assume that $\left(y_{1}, u_{1}\right)$ and $\left(y_{2}, u_{2}\right)$ are two different solutions of $(\mathcal{P})$. We note in particular that $y_{1} \neq y_{2}$ and we set $u=\left(u_{1}+u_{2}\right) / 2$. Looking at the proof of Proposition 3.4, we remark that one may prove the existence of a solution $y \in \mathcal{Y}$ of (1.1) corresponding to the control $u$, with $y \leq\left(y_{1}+y_{2}\right) / 2$. In fact we have that this inequality is strict in $\Omega$. Indeed

$$
-\Delta\left[\left(y_{1}+y_{2}\right) / 2-y\right] \geq \frac{1}{2}\left(\mathrm{e}^{y_{1}}+\mathrm{e}^{y_{2}}\right)-\mathrm{e}^{y} \geq \mathrm{e}^{\left(y_{1}+y_{2}\right) / 2}-\mathrm{e}^{y} \geq 0 \quad \text { in } \Omega
$$

and due to the fact that $y_{1} \neq y_{2}$, we have $\frac{1}{2}\left(\mathrm{e}^{y_{1}}+\mathrm{e}^{y_{2}}\right)-\mathrm{e}^{y} \neq 0$ on $\Omega$. Now by the strong maximum principle we conclude that $\left(y_{1}+y_{2}\right) / 2>y$ in $\Omega$. Therefore

$$
\begin{aligned}
\int_{\Omega} L(x, y(x)) d x & \leq \int_{\Omega} L\left(x,\left(y_{1}(x)+y_{2}(x)\right) / 2\right) d x \\
& \leq \frac{1}{2}\left(\int_{\Omega} L\left(x, y_{1}(x)\right) d x+\int_{\Omega} L\left(x, y_{2}(x)\right) d x\right)
\end{aligned}
$$


and the inequality

$$
\int_{\Omega} L(x, y(x)) d x \leq \frac{1}{2}\left(\int_{\Omega} L\left(x, y_{1}(x)\right) d x+\int_{\Omega} L\left(x, y_{2}(x)\right) d x\right)
$$

is strict if the strict convexity or monotonicity of $L(x, \cdot)$ is assumed. If the previous inequality is strict or if $N>0$ we deduce

$$
J(y, u)<\frac{1}{2}\left[J\left(y_{1}, u_{1}\right)+J\left(y_{2}, u_{2}\right)\right]=\inf (\mathcal{P}),
$$

which is a contradiction with the fact that $(u, y)$ is feasible for problem $(\mathcal{P})$.

\section{Existence of an Optimal Control. Case $p=2$}

As we noticed before, when $p=2$ the conclusions of Theorem 3.1 holds only when we know that $u_{k} \rightarrow u$ strongly in $L^{2}(\Omega)$. The difficulty comes from the fact that the strong convergence $\nabla y_{k} \rightarrow \nabla y$ can only be proved in $L^{r}(\Omega)$, with $r<2$. Hence we cannot pass to the limit in the inequality (2.8) satisfied for every $\left(y_{k}, u_{k}\right)$. Therefore we cannot deduce that $(y, u)$ satisfies (2.8), and consequently we are not able to prove the existence of a solution in $\mathcal{Y} \times K$. Since we have estimates on the state (see Theorem 2.5) only for elements of $\mathcal{Y}$, we cannot deduce, in general, the boundedness in $Y \times K$ of a minimizing sequence of problem $(P)$. In this section we will show that, under some additional assumptions on the function $L$, it is possible to have a minimizing sequence $\left\{\left(y_{k}, u_{k}\right)\right\}_{k=1}^{\infty}$ of $(\mathrm{P})$ with $\left\{y_{k}\right\}_{k=1}^{\infty} \subset \mathcal{Y}$. In this way, we can deduce the boundedness of the states and prove the existence of an optimal solution.

THEOREM 4.1. Let us assume that $\Omega$ is star-shaped with respect to one of its interior points. We also make the hypotheses

(i) The function $t \mapsto L(x, t)$ defined on $\mathbb{R}$ is monotone non decreasing for almost every $x \in \Omega$.

(ii) There exists a pair $(y, u) \in Y \times K$ satisfying (1.1).

(iii) Either $K$ is bounded or $N>0$ and $\theta_{2}<2$ in (1.4).

Then problems $(\mathrm{P})$ and $(\mathcal{P})$ have at least one solution. For each solution $(\bar{y}, \bar{u})$ of $(\mathrm{P})$, we can find $\tilde{y} \in \mathcal{Y}$ such that $(\tilde{y}, \bar{u})$ is a solution of $(\mathrm{P})$ and $(\mathcal{P})$. Moreover, if $L(x, \cdot)$ is strictly increasing for almost all $x \in \Omega$, then any optimal solution of $(\mathrm{P})$ is also a solution of $(\mathcal{P})$.

Proof. Let us take a minimizing sequence $\left\{\left(y_{k}, u_{k}\right)\right\}_{k=1}^{\infty} \subset Y \times K$. From Proposition 3.2 we deduce the existence of elements $z_{k} \in \mathcal{Y}$ such that $\left(z_{k}, u_{k}\right)$ satisfies (1.1). Moreover, by looking at the proof of the mentioned proposition, we know that $z_{k} \leq y_{k}$ in $\Omega$. Now using the monotonicity of $L(x, \cdot)$, we get that $J\left(z_{k}, u_{k}\right) \leq J\left(y_{k}, u_{k}\right)$. Therefore $\left\{\left(z_{k}, u_{k}\right)\right\}_{k=1}^{\infty}$ is also a minimizing sequence of $(\mathrm{P})$. Arguing as in the proof of Theorem 3.5, we can obtain a subsequence, denoted in the same way, converging to an element $(\bar{y}, \bar{u}) \in Y \times K$ solution of Problem (P).

If $\bar{y} \notin \mathcal{Y}$, we can apply again Proposition 3.2 to deduce the existence of an element $\tilde{y} \in \mathcal{Y}$, with $\tilde{y} \leq \bar{y}$, such that $(\tilde{y}, \bar{u})$ satisfies (1.1). Again the monotonicity of $L(x, \cdot)$ leads to $J(\tilde{y}, \bar{u}) \leq J(\bar{y}, \bar{u})$. So $(\tilde{y}, \bar{u})$ is a solution of $(\mathrm{P})$, and consequently of $(\mathcal{P})$ too. 
Finally, if $L(x, \cdot)$ is strictly increasing and $\bar{y} \notin \mathcal{Y}$, then $J(\tilde{y}, \bar{u})<J(\bar{y}, \bar{u})$, which contradicts the optimality of $(\bar{y}, \bar{u})$.

\section{The Optimality Conditions}

The aim of this section is to derive some optimality conditions for the control problem. We will prove two theorems corresponding to the cases $K=L^{p}(\Omega)$ and $K \subset L^{p}(\Omega)$ with $K \neq L^{p}(\Omega)$. Let us start with the first case.

Theorem 5.1. Assume that $\Omega$ is star-shaped with respect to one of its interior points, $p \geq 2$ and $K=L^{p}(\Omega)$. If $(\bar{y}, \bar{u})$ is a solution of problem $(\mathcal{P})$ (resp. $(\mathrm{P})$ ) with $\mathrm{e}^{\bar{y}} \in L^{p}(\Omega)$, then

$$
\begin{cases}-\Delta \bar{y}=\mathrm{e}^{\bar{y}}+\bar{u} & \text { in } \Omega, \\ \bar{y}=0 & \text { on } \Gamma,\end{cases}
$$

and setting $\bar{\phi}:=-N|\bar{u}|^{p-2} \bar{u}$, one has $\bar{\varphi} \in W_{0}^{1, s}(\Omega)$ for every $s<n /(n-1)$ and

$$
\begin{cases}-\Delta \bar{\varphi}=\mathrm{e}^{\bar{y}} \bar{\varphi}+\frac{\partial L}{\partial y}(x, \bar{y}) & \text { in } \Omega \\ \bar{\varphi}=0 & \text { on } \Gamma\end{cases}
$$

Moreover, if $p>n / 2, n \leq 5$ and $a_{1} \in L^{2 n /(n+2)}(\Omega)$ in $(1.3)$, then $\bar{\varphi} \in$ $H_{0}^{1}(\Omega)$.

Proof. Let us take $z \in H_{0}^{1}(\Omega) \cap L^{\infty}(\Omega)$ such that $\Delta z \in L^{p}(\Omega)$. For every $\lambda \in \mathbb{R}, \lambda \neq 0$, we set

$$
y_{\lambda}=\bar{y}+\lambda z \text { and } u_{\lambda}=\bar{u}-\lambda \Delta z+\mathrm{e}^{\bar{y}}-\mathrm{e}^{\bar{y}+\lambda z} .
$$

Then Corollary 2.4 implies that $y_{\lambda} \in \mathcal{Y}$, with $-\Delta y_{\lambda}=\mathrm{e}^{y_{\lambda}}+u_{\lambda}$. On the other hand $u_{\lambda} \in L^{p}(\Omega)$. Then $\left(y_{\lambda}, u_{\lambda}\right)$ is a feasible point for $(\mathcal{P})$ (resp. $(\mathrm{P})$ ), consequently, using Lebesgue's convergence theorem along with assumption (1.3), we get

$$
\begin{aligned}
0 & \leq \lim _{\lambda \rightarrow 0} \frac{J\left(y_{\lambda}, u_{\lambda}\right)-J(\bar{y}, \bar{u})}{\lambda} \\
& =\lim _{\lambda \rightarrow 0} \int_{\Omega} \frac{L\left(x, y_{\lambda}(x)\right)-L(x, \bar{y}(x))}{\lambda} d x+\lim _{\lambda \rightarrow 0} \frac{N}{p} \int_{\Omega} \frac{\left|u_{\lambda}(x)\right|^{p}-|\bar{u}(x)|^{p}}{\lambda} d x \\
& =\int_{\Omega} \frac{\partial L}{\partial y}(x, \bar{y}(x)) z(x) d x+N \int_{\Omega}|\bar{u}|^{p-2} \bar{u}\left(-\Delta z-\mathrm{e}^{\bar{y}} z\right) d x
\end{aligned}
$$

From the linearity of the previous relation with respect to $z$ we deduce that

$$
\int_{\Omega} \frac{\partial L}{\partial y}(x, \bar{y}(x)) z(x) d x+N \int_{\Omega}|\bar{u}|^{p-2} \bar{u}\left(-\Delta z-\mathrm{e}^{\bar{y}} z\right) d x=0
$$

for every $z \in H_{0}^{1}(\Omega) \cap L^{\infty}(\Omega)$ such that $\Delta z \in L^{p}(\Omega)$. Let us set $\bar{\varphi}:=$ $-N|\bar{u}|^{p-2} \bar{u}$, then

$$
\int_{\Omega} \bar{\varphi}\left(-\Delta z-\mathrm{e}^{\bar{y}} z\right) d x=\int_{\Omega} \frac{\partial L}{\partial y}(x, \bar{y}(x)) z(x) d x
$$


Given $f \in C_{0}^{\infty}(\Omega)$, let $z \in H_{0}^{1}(\Omega)$ be the solution of Dirichlet problem

$$
\begin{cases}-\Delta z=f & \text { in } \Omega, \\ z=0 & \text { on } \Gamma .\end{cases}
$$

Then $z \in H_{0}^{1}(\Omega) \cap L^{\infty}(\Omega)$ and for $s<n /(n-1)$ and $(1 / s)+\left(1 / s^{\prime}\right)=1$ we have (see G. Stampacchia [12], Theorems 4.1 and 4.2)

$$
\|z\|_{L^{\infty}(\Omega)} \leq C_{s}\|f\|_{W^{-1, s^{\prime}(\Omega)}}
$$

Combining (5.3), (5.4) and (5.5) we get

$$
\begin{aligned}
\int_{\Omega} \bar{\varphi} f d x & =\int_{\Omega} \bar{\varphi}(-\Delta z) d x=\int_{\Omega}\left\{\frac{\partial L}{\partial y}(x, \bar{y}(x)) z(x)+\mathrm{e}^{\bar{y}} z\right\} d x \\
& \leq\left(\left\|\frac{\partial L}{\partial y}(x, \bar{y})\right\|_{L^{1}(\Omega)}+\left\|\mathrm{e}^{\bar{y}}\right\|_{L^{1}(\Omega)}\right)\|z\|_{L^{\infty}(\Omega)} \\
& \leq C_{s}\left(\left\|\frac{\partial L}{\partial y}(x, \bar{y})\right\|_{L^{1}(\Omega)}+\left\|\mathrm{e}^{\bar{y}}\right\|_{L^{1}(\Omega)}\right)\|f\|_{W^{-1, s^{\prime}}(\Omega)}
\end{aligned}
$$

Taking into account that $C_{0}^{\infty}(\Omega)$ is dense in $W^{-1, s^{\prime}}(\Omega)$, we deduce from the above inequality that $\bar{\varphi} \in\left(W^{-1, s^{\prime}}(\Omega)\right)^{\prime}=W_{0}^{1, s}(\Omega)$ for every $s<n /(n-1)$. The fact that (5.2) follows from (5.3) is a straightforward consequence of the definition of $\bar{\varphi}$.

Finally, if $p>n / 2$, from the fact that $-\Delta \bar{y}=\mathrm{e}^{\bar{y}}+\bar{u} \in L^{p}(\Omega)$ and using again the above mentioned results of $G$. Stampacchia [12], it follows that $\bar{y} \in L^{\infty}(\Omega)$. On the other hand, $W_{0}^{1, s}(\Omega) \subset L^{n s /(n-s)}(\Omega) \subset H^{-1}(\Omega)$ if $s$ is close enough to $n /(n-1)$ and $n \leq 5$. Therefore the right hand side of $(5.2)$ belongs to $H^{-1}(\Omega)$, which allows to conclude that $\bar{\varphi} \in H_{0}^{1}(\Omega)$.

In case of a problem with control constraints, we have the following result. THEOREM 5.2. Let us assume that $\Omega$ is star-shaped with respect to some $x_{0} \in \Omega, p \geq 2$ and $p>n / 2$. If $(\bar{y}, \bar{u})$ is a solution of problem $(\mathcal{P})$ (resp. $(\mathrm{P})$ ) with $\mathrm{e}^{\bar{y}} \in L^{p}(\Omega)$, then there exist a real number $\bar{\alpha} \geq 0$ and a function $\bar{\varphi} \in W_{0}^{1, s}(\Omega)$ for every $s<n /(n-1)$ such that

$$
\begin{gathered}
\bar{\alpha}+\|\bar{\varphi}\|_{W_{0}^{1, s}(\Omega)}>0 ; \\
\begin{cases}-\Delta \bar{y}=\mathrm{e}^{\bar{y}}+\bar{u} & \text { in } \Omega, \\
\bar{y}=0 & \text { on } \Gamma ;\end{cases} \\
\begin{cases}-\Delta \bar{\varphi}=\mathrm{e}^{\bar{y}} \bar{\varphi}+\bar{\alpha} \frac{\partial L}{\partial y}(x, \bar{y}) & \text { in } \Omega, \\
\bar{\varphi}=0 & \text { on } \Gamma ;\end{cases} \\
\int_{\Omega}\left(\bar{\varphi}+\bar{\alpha} N|\bar{u}|^{p-2} \bar{u}\right)(u-\bar{u}) d x \geq 0 \quad \forall u \in K .
\end{gathered}
$$

Moreover, if $n \leq 5$ and $a_{1} \in L^{2 n /(n+2)}(\Omega)$ in $(1.3)$, then $\bar{\varphi} \in H_{0}^{1}(\Omega)$. ESAIM: COCV, November 1998, Vol. 3, 361-380 
The proof of this theorem requires some previous lemmas. First of all, let us remark that $\bar{y} \in L^{\infty}(\Omega)$. Indeed $-\Delta \bar{y}=\mathrm{e}^{\bar{y}}+\bar{u} \in L^{p}(\Omega)$ with $p>n / 2$, thus it is enough to use again the mentioned results of G. Stampacchia [12] to deduce the boundedness of $\bar{y}$. In particular we have that if $(\bar{y}, \bar{u})$ is a solution of $(\mathrm{P})$, then it is also a solution of $(\mathcal{P})$ because $\bar{y} \in \mathcal{Y}$; see Corollary 2.4 .

Given $\epsilon>0$ we define $J_{\epsilon}: L^{p}(\Omega) \times L^{p}(\Omega) \longrightarrow \mathbb{R}$ by

$$
\begin{aligned}
J_{\epsilon}(u, w) & :=J\left(y_{(u, w)}, u\right)+\frac{1}{p \epsilon} \int_{\Omega}\left|\mathrm{e}^{y_{(u, w)}}-w\right|^{p} d x \\
& +\frac{1}{p} \int_{\Omega}|u-\bar{u}|^{p} d x+\frac{1}{p} \int_{\Omega}\left|\mathrm{e}^{\bar{y}}-w\right|^{p} d x,
\end{aligned}
$$

where $y_{(u, w)}$ is the unique element of $H_{0}^{1}(\Omega)$ solving the boundary value problem

$$
\begin{cases}-\Delta y=u+w & \text { in } \Omega, \\ y=0 & \text { on } \Gamma .\end{cases}
$$

Once again using the mentioned results of G. Stampacchia [12] we obtain that $y_{(u, u)} \in L^{\infty}(\Omega)$ and therefore $J_{\epsilon}$ is well defined. Now we consider the following control problem

$$
\left(\mathrm{P}_{\epsilon}\right)\left\{\begin{array}{l}
\text { Minimize } J_{\epsilon}(u, w) \\
(u, w) \in K \cap \bar{B}_{1}(\bar{u}) \times \bar{B}_{1}\left(\mathrm{e}^{\bar{y}}\right),
\end{array}\right.
$$

where $\bar{B}_{1}(\bar{u})$ (resp. $\bar{B}_{1}\left(\mathrm{e}^{\bar{y}}\right)$ ) denotes the closed unit ball of $L^{p}(\Omega)$ with center at $\bar{u}$ (resp. $\mathrm{e}^{\bar{y}}$ ). We have the following result.

Lemma 5.3. Problem $\left(\mathrm{P}_{\epsilon}\right)$ has at least one solution $\left(u_{\epsilon}, w_{\epsilon}\right)$. Moreover we have

$$
\begin{gathered}
\lim _{\epsilon \rightarrow 0}\left\|u_{\epsilon}-\bar{u}\right\|_{L^{p}(\Omega)}=\lim _{\epsilon \rightarrow 0}\left\|w_{\epsilon}-\mathrm{e}^{\bar{y}}\right\|_{L^{p}(\Omega)}=\lim _{\epsilon \rightarrow 0} \frac{1}{\epsilon}\left\|\mathrm{e}^{y_{\epsilon}}-w_{\epsilon}\right\|_{L^{p}(\Omega)}^{p}=0 \\
\lim _{\epsilon \rightarrow 0}\left\|y_{\epsilon}-\bar{y}\right\|_{L^{\infty}(\Omega)}=\lim _{\epsilon \rightarrow 0}\left\|y_{\epsilon}-\bar{y}\right\|_{H_{0}^{1}(\Omega)}=0
\end{gathered}
$$

where $y_{\epsilon}$ is the solution of (5.10) corresponding to $\left(u_{\epsilon}, w_{\epsilon}\right)$.

Proof. The existence of a solution is obvious because of the convexity, boundedness and closedness of the set of feasible controls as well as the weak lower semicontinuity of $J_{\epsilon}$. Furthermore $\left\{\left(u_{\epsilon}, w_{\epsilon}\right)\right\}_{0<\epsilon<1}$ is bounded in $L^{p}(\Omega) \times$ $L^{p}(\Omega)$, consequently $\left\{y_{\epsilon}\right\}_{0<\epsilon<1}$ is bounded in $H_{0}^{1}(\Omega) \cap L^{\infty}(\Omega)$. Then we can take subsequences such $\epsilon_{k} \searrow 0$ as $k \rightarrow \infty$ and $\left(u_{\epsilon_{k}}, w_{\epsilon_{k}}\right) \rightarrow(\tilde{u}, \tilde{w})$ weakly in $L^{p}(\Omega) \times L^{p}(\Omega)$. Hence we get from (5.10) that $y_{\epsilon_{k}} \rightarrow \tilde{y}=y_{(\tilde{u}, \tilde{w})}$ strongly in $H_{0}^{1}(\Omega) \cap L^{\infty}(\Omega)$.

Since $\left(\bar{u}, \mathrm{e}^{\bar{y}}\right)$ is a feasible control for $\left(\mathrm{P}_{\epsilon}\right)$ and $\bar{y}=y_{\left(\bar{u}, \mathrm{e}^{\bar{y}}\right)}$, we have that $J_{\epsilon}\left(u_{\epsilon}, w_{\epsilon}\right) \leq J_{\epsilon}\left(\bar{u}, \mathrm{e}^{\bar{y}}\right)=J(\bar{y}, \bar{u})$. Then we have

$$
\lim _{\epsilon \rightarrow 0}\left\|\mathbf{e}^{y_{\epsilon}}-w_{\epsilon}\right\|_{L^{p}(\Omega)}=0
$$

which implies that $\mathrm{e}^{\tilde{y}}=\tilde{w}$. Therefore $\tilde{y} \in \mathcal{Y}$ is a solution of (1.1) corresponding to $\tilde{u}$. So $(\tilde{y}, \tilde{u})$ is a feasible point for problem (P) (and also for 
$(\mathcal{P})$ ), hence

$$
\begin{aligned}
J(\bar{y}, \bar{u}) & \leq J(\tilde{y}, \tilde{u}) \leq J(\tilde{y}, \tilde{u})+\frac{1}{p}\|\tilde{u}-\bar{u}\|_{L^{p}(\Omega)}^{p}+\frac{1}{p}\left\|\mathrm{e}^{\bar{y}}-\mathrm{e}^{\tilde{y}}\right\|_{L^{p}(\Omega)}^{p} \\
& \leq \liminf _{k \rightarrow \infty} J_{\epsilon_{k}}\left(u_{\epsilon_{k}}, w_{\epsilon_{k}}\right) \leq J(\bar{y}, \bar{u})
\end{aligned}
$$

which leads to the equalities $\tilde{u}=\bar{u}$ and $\tilde{y}=\bar{y}$. Now the convergences (5.11) and (5.12) follow from the above inequality and the state equation (5.10).

Lemma 5.4. There exist $\epsilon_{0}>0$ and a sequence $\left\{\left(u_{\epsilon}, w_{\epsilon}\right)\right\}_{0<\epsilon<\epsilon_{0}}$ such that $\left(u_{\epsilon}, w_{\epsilon}\right)$ is a solution of $\left(\mathrm{P}_{\epsilon}\right)$ and for all $\epsilon>0$ such that $\epsilon<\epsilon_{0}$ :

$$
\left\|\bar{u}-u_{\epsilon}\right\|_{L^{p}(\Omega)}<1 \quad \text { and } \quad\left\|\bar{u}-u_{\epsilon}\right\|_{L^{p}(\Omega)}<1 .
$$

Furthermore, there exists $\varphi_{\epsilon} \in W_{0}^{1, s}(\Omega)$ for every $s<n /(n-1)$ such that

$$
\begin{gathered}
\begin{cases}-\Delta y_{\epsilon}=u_{\epsilon}+w_{\epsilon} & \text { in } \Omega, \\
y_{\epsilon}=0 & \text { on } \Gamma ;\end{cases} \\
\begin{cases}-\Delta \varphi_{\epsilon}=\mathrm{e}^{y_{\epsilon}} \varphi_{\epsilon}+\frac{\partial L}{\partial y}\left(x, y_{\epsilon}\right)+g_{\epsilon} & \text { in } \Omega, \\
\varphi_{\epsilon}=0 & \text { on } \Gamma ;\end{cases}
\end{gathered}
$$

$\forall u \in K, \quad \int_{\Omega}\left\{\varphi_{\epsilon}+N\left|u_{\epsilon}\right|^{p-2} u_{\epsilon}+\left|u_{\epsilon}-\bar{u}\right|^{p-2}\left(u_{\epsilon}-\bar{u}\right)\right\}\left(u-u_{\epsilon}\right) d x \geq 0$,

where $g_{\epsilon} \rightarrow 0$ in $L^{p^{\prime}}(\Omega)$ as $\epsilon \searrow 0$.

Proof. The existence of a sequence $\left\{\left(u_{\epsilon}, w_{\epsilon}\right)\right\}_{0<\epsilon<\epsilon_{0}}$ of solutions satisfying (5.13) is a consequence of Lemma 5.3. Given $h \in L^{p}(\Omega)$ we denote by $z_{h} \in H_{0}^{1}(\Omega) \cap L^{\infty}(\Omega)$ the solution of

$$
\begin{cases}-\Delta z=h & \text { in } \Omega \\ z=0 & \text { on } \Gamma\end{cases}
$$

Let us set $y_{\epsilon}=y_{\left(u_{\epsilon}, w_{\epsilon}\right)}$ and

$$
\varphi_{\epsilon}=\frac{1}{\epsilon}\left|\mathrm{e}^{y_{\epsilon}}-w_{\epsilon}\right|^{p-2}\left(\mathrm{e}^{y_{\epsilon}}-w_{\epsilon}\right)+\left|\mathrm{e}^{\bar{y}}-w_{\epsilon}\right|^{p-2}\left(\mathrm{e}^{\bar{y}}-w_{\epsilon}\right) .
$$

From the optimality of $\left(u_{\epsilon}, w_{\epsilon}\right)$ we deduce that for all $u \in K$ and $w \in L^{p}(\Omega)$ :

$$
\frac{\partial J_{\epsilon}}{\partial u}\left(u_{\epsilon}, w_{\epsilon}\right)\left(u-u_{\epsilon}\right) \geq 0 \quad \text { and } \quad \frac{\partial J_{\epsilon}}{\partial w}\left(u_{\epsilon}, w_{\epsilon}\right) w=0 .
$$

We compute the second derivative for each $w \in L^{p}(\Omega)$

$$
\frac{\partial J_{\epsilon}}{\partial w}\left(u_{\epsilon}, w_{\epsilon}\right) w=\int_{\Omega}\left\{\frac{\partial L}{\partial y}\left(x, y_{\epsilon}\right)+\mathrm{e}^{y_{\epsilon}} \varphi_{\epsilon}+g_{\epsilon}\right\} z_{w} d x-\int_{\Omega} \varphi_{\epsilon} w d x=0
$$

where

$$
g_{\epsilon}=-\mathrm{e}^{y_{\epsilon}}\left|\mathrm{e}^{\bar{y}}-w_{\epsilon}\right|^{p-2}\left(\mathrm{e}^{\bar{y}}-w_{\epsilon}\right) .
$$

From (5.11) and (5.12) we get that $g_{\epsilon} \rightarrow 0$ strongly in $L^{p^{\prime}}(\Omega)$. 
Computing now the first derivative of $(5.17)$

$$
\begin{gathered}
\frac{\partial J_{\epsilon}}{\partial u}\left(u_{\epsilon}, w_{\epsilon}\right)\left(u-u_{\epsilon}\right)=\int_{\Omega}\left\{\frac{\partial L}{\partial y}\left(x, y_{\epsilon}\right)+\mathrm{e}^{y_{\epsilon}} \varphi_{\epsilon}+g_{\epsilon}\right\} z_{u-u_{\epsilon}} d x \\
+\int_{\Omega}\left\{N\left|u_{\epsilon}\right|^{p-2} u_{\epsilon}+\left|u_{\epsilon}-\bar{u}\right|^{p-2}\left(u_{\epsilon}-\bar{u}\right)\right\}\left(u-u_{\epsilon}\right) d x \geq 0 .
\end{gathered}
$$

Taking $w=u-u_{\epsilon}$ in (5.18), one sees that (5.16) follows from this inequality. Finally, from (5.18) we get that

$$
\int_{\Omega} \varphi_{\epsilon}(-\Delta z) d x=\int_{\Omega}\left\{\frac{\partial L}{\partial y}\left(x, y_{\epsilon}\right)+\mathrm{e}^{y_{\epsilon}} \varphi_{\epsilon}+g_{\epsilon}\right\} z d x
$$

for every $z \in H_{0}^{1}(\Omega)$ with $-\Delta z \in L^{p}(\Omega)$. Arguing as in the proof of Theorem 5.1 , we deduce from here that $\varphi_{\epsilon} \in W_{0}^{1, s}(\Omega)$ for every $s<n /(n-1)$ and (5.15) holds.

Now we are ready to conclude the proof of Theorem 5.2.

Proof of Theorem 5.2. If $\left\{\varphi_{\epsilon}\right\}_{0<\epsilon<\epsilon_{0}}$ is bounded in $L^{1}(\Omega)$, then $\left\{\varphi_{\epsilon}\right\}_{0<\epsilon<\epsilon_{0}}$ is also bounded in $W_{0}^{1, s}(\Omega)$ and it is easy to pass to the limit in $(5.14)-(5.16)$ with the aid of Lemma 5.3 and to deduce $(5.7)-(5.9)$ with $\bar{\alpha}=1$. Otherwise we take

$$
\alpha_{\epsilon}=\frac{1}{\left\|\varphi_{\epsilon}\right\|_{L^{1}(\Omega)}}
$$

and we redefine $\varphi_{\epsilon}$ as $\alpha_{\epsilon} \varphi_{\epsilon}$. Then (5.15) and (5.16) can be written

$$
\begin{cases}-\Delta \varphi_{\epsilon}=\mathrm{e}^{y_{\epsilon}} \varphi_{\epsilon}+\alpha_{\epsilon} \frac{\partial L}{\partial y}\left(x, y_{\epsilon}\right)+\alpha_{\epsilon} g_{\epsilon} & \text { in } \Omega \\ \varphi_{\epsilon}=0 & \text { on } \Gamma\end{cases}
$$

and

$\forall u \in K, \quad \int_{\Omega}\left\{\varphi_{\epsilon}+\alpha_{\epsilon} N\left|u_{\epsilon}\right|^{p-2} u_{\epsilon}+\alpha_{\epsilon}\left|u_{\epsilon}-\bar{u}\right|^{p-2}\left(u_{\epsilon}-\bar{u}\right)\right\}\left(u-u_{\epsilon}\right) d x \geq 0$,

respectively. Now $\left\{\varphi_{\epsilon}\right\}_{0<\epsilon<\epsilon_{0}}$ is bounded in $L^{1}(\Omega)$ and $\alpha_{\epsilon} \rightarrow 0$, then we can pass to the limit in the previous relations and to obtain (5.8) and (5.9) with $\bar{\alpha}=0$. It remains to prove that (5.6) holds, or equivalently that $\bar{\varphi} \neq 0$. From the equation satisfied by $\varphi_{\epsilon}$ we deduce that $\left\{\varphi_{\epsilon}\right\}_{0<\epsilon<\epsilon_{0}}$ is bounded in $W_{0}^{1, s}(\Omega)$ for every $s<n /(n-1)$. Then we can take a subsequence, denoted in the same way, such that $\varphi_{\epsilon} \rightarrow \bar{\varphi}$ weakly in $W_{0}^{1, s}(\Omega)$, hence also strongly in $L^{1}(\Omega)$. Now the equality $\left\|\varphi_{\epsilon}\right\|_{L^{1}(\Omega)}=1$ leads to $\|\bar{\varphi}\|_{L^{1}(\Omega)}=1$.

The $H_{0}^{1}(\Omega)$-regularity of $\bar{\varphi}$ claimed in the theorem follows as in the proof of Theorem 5.1 .

In some cases we can prove that $\bar{\alpha}$ can be chosen equal to one in the system (5.7)-(5.9)

Corollary 5.5. Under the assumptions of Theorem 5.2, if furthermore there exists an open set $\omega \subset \Omega$ and a neighborhood $\mathcal{W}$ of zero in $C_{0}^{\infty}(\omega)$ such that $\bar{u}+w \in K$ for every $w \in \mathcal{W}$, then (5.7) -(5.9) holds with $\bar{\alpha}=1$. 
Proof. If $\bar{\alpha}=0$ in (5.7)-(5.9), then from the hypothesis of the corollary and (5.9) we deduce that $\bar{\varphi}(x)=0$ for almost all $x \in \omega$. Now (5.8) is

$$
\begin{cases}-\Delta \bar{\varphi}=\mathrm{e}^{\bar{y}} \bar{\varphi} & \text { in } \Omega . \\ \bar{\varphi}=0 & \text { on } \Gamma .\end{cases}
$$

Then we deduce that $\bar{\varphi}=0$ in $\Omega$ (see J.C. Saut and B. Scheurer [11]), which contradicts (5.6).

\section{REFERENCES}

[1] H. Amann: On the number of solutions of nonlinear equations in ordered Banach spaces. J. Func. Anal., 11 1972, 346-384.

[2] L. Boccardo, F. Murat: Almost everywhere convergence of the gradients of solutions to elliptic and parabolic equations. Nonlinear Anal., Theory, Methods, Appl., 19 1992, $581-597$.

[3] S. Chandrasekhar: An introduction to the study of stellar structures. Dover Publishing Inc., 1985.

[4] M.G. Crandall, P.H. Rabinowitz: Some continuation and variational methods for positive solutions of nonlinear elliptic eigenvalue problems. Arch. Rational Mech. Anal, $\mathbf{5 8} 1975,207-218$.

[5] D.A. Franck-Kamenetskii: Diffusion and heat transfer in chemical kinetics. Second edition, Plenum Press, 1969.

[6] Th. Gallouët, F. Mignot, J.P. Puel: Quelques résultats sur le problème $-\Delta u=\lambda e^{u}$. C. R. Acad. Sci. Paris, 307, série I, 1988, 289-292.

[7] I.M. Gelfand: Some problems in the theory of quasi-linear equations. Uspekhi Mat. Nauk, (N.S.), 14 (86), 1959, 87-158 (in russian); Amer. Math. Soc. Transl., (Ser. 2), 29, 1963, 289-292.

[8] F. Mignot, J.P. Puel: Sur une classe de problèmes non linéaires avec nonlinéarité positive, croissante, convexe. Comm. PDE, 5 (8), 1980, 791-836.

[9] F. Mignot, J.P. Puel: Solution singulière radiale de $-\Delta u=\lambda e^{u}$. C. R. Acad. Sci. Paris, 307, série I, 1988, 379-382.

[10] D.H. Sattinger: Monotone methods in nonlinear elliptic and parabolic boundary value problems. Indiana Univ. Math. J., 21 1972, 979-1000.

[11] J.C. Saut, B. Scheurer: Sur l'unicité du problème de cauchy et le prolongement unique pour des équations elliptiques à coefficients non localement bornés. J. Diff. Eq., 43 $1982,28-43$.

[12] G. Stampacchia: Le problème de Dirichlet pour les équations elliptiques du second ordre à coefficients discontinus. Ann. Inst. Fourier, 15 1965, 189-258. 